\title{
Artigo
}

\section{Término de relacionamentos íntimos violentos: uma revisão da literatura}

\author{
Sheila Giardini Murta* (1) \\ Priscila de Oliveira Parada (1) \\ Universidade de Brasília, Brasília, DF, Brasil
}

\begin{abstract}
Resumo: Este artigo analisa avanços e limitações na produção científica acerca do processo de término de relações íntimas violentas. Realizou-se busca nas bases de dados Portal de Periódicos Capes e SciELO, com entradas em inglês, francês, espanhol e português. O critério de inclusão foi tratar do término de relações amorosas violentas ou de aspectos da decisão ficar versus sair. Encontraram-se catorze estudos publicados entre 1999 e 2015, dos quais metade tinha design quantitativo e a outra metade, qualitativo. Estas variáveis mostraram correlação com o avanço rumo ao término nos estudos quantitativos: nível de investimento, comprometimento, normas subjetivas, atribuir ao agressor a responsabilidade pela agressão, sentimento de raiva, barreiras e facilitadores estruturais. Para os sete estudos qualitativos, observaram-se: a proposta de novos modelos de compreensão, a maior variabilidade de recrutamento das amostras e a consideração do período pós-separação nas análises. Discutem-se recomendações para a prática profissional e a pesquisa.
\end{abstract}

Palavras-chave: violência doméstica, violência por parceiro íntimo, violência contra a mulher.

Estudos acerca da prevalência e dos impactos da violência nos relacionamentos íntimos são crescentes e têm se tornado cada vez mais importantes, tanto na compreensão desse fenômeno como na construção de diretrizes das políticas públicas relacionadas ao tema (Brasil, 2006). Dados sobre a prevalência apontam que ao menos uma em cada cinco mulheres brasileiras sofrem com a violência nos relacionamentos (DataSenado, 2013) e que $43 \%$ das mulheres que buscam ajuda via assistência telefônica são agredidas diariamente e $35 \%$, semanalmente (Secretaria Especial de Políticas para as Mulheres, 2012). Esses dados, contudo, ainda devem ser tratados como limites mínimos, uma vez que é comum que tanto mulheres como homens não saibam reconhecer formas de violência diversas da agressão física.

Nesse contexto, os estudos sobre término de relacionamentos violentos apresentam potencial de maior esclarecimento sobre os processos que propiciam o fim desse tipo de relação. Ampliar a compreensão desse processo complexo poderá gerar conclusões relevantes e aplicáveis às políticas públicas e aos serviços de prevenção - no sentido do abandono precoce de relações que se mostrem violentas - e de suporte a vítimas de violência.

A superação da condição de violência no relacionamento engloba mais de um caminho possível. Uma primeira variante seria a interrupção da violência dentro do próprio relacionamento. Outra possibilidade consistiria no término da relação violenta. Sobre a primeira opção, contudo, revisões sistemáticas e metanálises têm apontado para a inconsistência dos resultados de

* Endereço para correspondência: giardini@unb.br intervenções realizadas com homens autores de violência encaminhados pela justiça (Akoensi, Koehler, Lösel, \& Humphreys, 2013; Feder \& Wilson 2005; Feder, Wilson, \& Austin, 2008). As revisões da literatura sobre o tema (Feder \& Wilson 2005; Feder et al., 2008) mostraram que estudos que utilizaram métodos experimentais para avaliação de efeitos das referidas intervenções apresentaram efeitos modestos quando comparados os grupos de tratamento e controle. Por sua vez, aqueles de delineamento quase-experimental com grupo de comparação sem tratamento indicaram resultados inconsistentes, com possibilidades de pequenos efeitos prejudiciais. As evidências, portanto, apontam para o fraco efeito das intervenções direcionadas aos agressores existentes atualmente. Por um lado, isso suscita a necessidade de investigações que permitam o desenvolvimento de técnicas e intervenções mais efetivas e, por outro, que as intervenções destinadas à superação da violência enfoquem tanto as mudanças de comportamento do agressor quanto a possibilidade de abandono da relação pela vítima.

Nesse sentido, resta analisar as possibilidades relacionadas ao outro caminho possível: o término do relacionamento. Essa, contudo, não se mostra uma via simples, envolvendo outras tantas ramificações de possibilidades no que diz respeito à continuidade versus superação da violência. Em primeiro lugar, sabe-se que o término não necessariamente significa o fim da violência e, em alguns casos, a avaliação dos riscos diante de uma ou outra opção pela vítima leva-a a concluir que a permanência, ao menos no curto prazo, é a opção mais segura (Anderson, 2003; Bell, Goodman, \& Dutton, 2007; Meyer, 2012). 
Em segundo, os resultados de alguns estudos demonstraram que o término da relação não necessariamente é definitivo (Bell et al., 2007; Shurman \& Rodrigues, 2007) e, ainda, que nem sempre é acompanhado de bem-estar. Shurman e Rodriguez (2007), nesse sentido, encontraram a depressão como aspecto emocional associado ao período que segue o término da relação. Esses resultados apontam para a necessidade de estudos que clarifiquem quais outros fatores contribuem para a melhoria da qualidade de vida de pessoas que conseguem sair de relações violentas e da revisão das políticas públicas, cuja atuação não se encerra quando ocorre a separação.

Em terceiro lugar, existe a possibilidade do engajamento em novas relações violentas após o abandono da anterior. Martsof, Draucker, Stephenson, Cook e Heckman (2012), em um estudo qualitativo envolvendo 88 participantes, encontraram que 32 haviam vivido mais de um namoro violento no período da adolescência. Isso ressalta o fato de que a superação do ciclo da violência é um fenômeno complexo e ainda pouco conhecido que merece muita atenção.

Embora o abandono do relacionamento não se constitua em todos os casos como uma opção viável ao menos no curto prazo - ou não implique necessariamente o fim da violência, ele segue sendo um caminho possível, direcionado ao empoderamento e cuja compreensão tem potencial de aprimorar as políticas de atendimento a vítimas de agressões por parceiros íntimos, seja pela oferta de novos serviços voltados a necessidades que atualmente não são atendidas ou não entraram na agenda, pelo desenvolvimento de perspectivas preventivas, especialmente da prevenção indicada (aquela direcionada a pessoas que já apresentam um problema em estágio inicial), ou pela qualificação de técnicas e métodos de intervenção mais eficazes nos serviços já existentes.

No único artigo anterior encontrado de revisão de literatura sobre o tema do abandono de relações violentas, Anderson e Saunders (2003) ressaltaram a consistência de variáveis e as tendências na agenda de pesquisa em 20 estudos quantitativos e 28 estudos qualitativos publicados entre 1976 e 2001. No que diz respeito aos estudos quantitativos, essa revisão identificou a existência de quatro grupos de variáveis sobre as quais os estudos se concentraram: frequência e severidade da violência, história de vitimização da mulher na infância ou família de origem, fatores psicossociais, recursos externos e estratégias de enfrentamento anteriores. Sobre a relação entre frequência e severidade da violência e término da relação, os estudos mostraram-se inconsistentes, provavelmente em razão de conceituação da severidade e de medidas utilizadas. O mesmo ocorreu no que diz respeito à vivência de violência na infância ou família de origem, tendo sido os resultados entre os estudos inconsistentes para apontar esse como um fator que facilite ou dificulte o abandono da relação.

Sobre os fatores psicossociais, o mais estudado nas pesquisas incluídas na revisão de Anderson e
Saunders (2003) consistiu no conceito de comprometimento com o relacionamento, hipotetizando que mulheres com maior comprometimento tenderiam a permanecer mais no relacionamento. Essa relação foi observada quando se utilizaram medidas subjetivas (ex.: sentimentos positivos em relação ao parceiro), contudo mostrou-se mais fraca diante de medidas objetivas (ex.: tempo e status do relacionamento). As variáveis ligadas a recursos externos, especialmente aquelas relacionadas à renda, por sua vez, foram as que se apresentaram mais consistentes e tiveram maior poder de predição ao longo dos estudos. Nesse sentido, menos acesso a renda própria ou a outros recursos externos dificultou o término da relação.

Por fim, os estudos que inseriram variáveis relacionadas às estratégias de enfrentamento da violência são apontados por Anderson e Saunders (2003) como precursores da ideia do abandono enquanto processo. Essas pesquisas evidenciaram que mulheres com maior número de tentativas de término definitivo foram as mais aptas a alcançá-lo e que aquelas que empregaram múltiplas estratégias de enfrentamento, além do rompimento, tinham maiores chances de conseguirem se separar. A autora e o autor indicam, ainda, a mudança proporcionada por essas pesquisas no foco de interesse, que deixa de residir nos motivos pelos quais as mulheres permanecem na relação para aqueles que permitem que elas as deixem (Anderson \& Saunders, 2003).

No que diz respeito às investigações qualitativas, Anderson e Saunders (2003) apontaram para maior tendência, nesses estudos, de conceituar o abandono como um processo que não se restringe ao término definitivo, mas envolve decisões e ações que ocorrem durante meses ou anos. Os pesquisadores interpretam o fenômeno, portanto, como esforços ativos e cumulativos pelos quais as mulheres aprendem gradualmente estratégias mais efetivas para lidar com o abuso, incluindo o abandono. O modelo transteórico de mudança (Prochaska, DiClemente, \& Norcross, 1984) destacou-se como referencial de interpretação dos dados qualitativos entre os estudos incluídos na revisão. Eles identificaram que as mulheres passaram por um aumento de suas perspectivas que, em alguns casos, ocorreu repentinamente, mas, na maioria, desenvolveram-se a partir de percepções fugidias sobre a relação e sobre si. Como catalizadores no processo de abandono da relação, as pesquisas qualitativas englobadas pela revisão encontraram aumento no nível da violência, menor quantidade de carinho e dedicação pelo parceiro, perda da esperança na melhoria da relação, perceber o efeito do abuso nos filhos e a rede de suporte social comunitária e profissional.

Anderson e Saunders (2003) destacam, ainda, aspectos relacionados ao bem-estar psicológico após a separação. Essa foi uma lacuna identificada por eles naquele momento da produção científica. Entre os poucos estudos analisados por eles que se preocuparam com isso, os resultados mostraram que, na medida em que passa o tempo desde a separação, aumenta o bem-estar psicológico. 
Por outro lado, quando considerados grupos distintos ao invés de médias gerais, encontrou-se prevalência considerável de sintomas de depressão, estresse pós-traumático e ansiedade após o término da relação violenta, o que chama atenção para a necessidade de maior compreensão sobre esse período para tais grupos e da investigação sobre eventuais fragilidades sociodemográficas, de saúde ou de suporte social associadas a esses desfechos.

Este artigo tem como objetivo analisar os avanços, as limitações e as lacunas nas pesquisas publicadas após a revisão anterior (Anderson \& Saunders, 2003) ou não inseridas nela acerca do abandono de relacionamentos íntimos violentos e suas implicações. Definem-se relacionamentos íntimos como relacionamentos afetivosexuais pré-maritais ou maritais. Considera-se que tal esforço poderá contribuir para uma melhor compreensão do estado da arte acerca do tema cujo aprofundamento permitirá o desenvolvimento e revisão de direcionamentos tanto para o tratamento quanto para a prevenção indicada em situações de violência por parceiro íntimo.

\section{Método}

Realizou-se busca nos portais Periódicos Capes e SciELO, utilizando-se combinações de palavras em inglês, português, espanhol e francês: abandonar, terminar, sair, deixar, namoros, relacionamentos, relação, casamento, violento(as), violência por parceiro íntimo (português), leave, stay/leave, abandon, end, date, relationship, IPV, partner, violence, violent, aggressive (inglês), dejar, salir, acabar, romper, relación, noviazgo, violento(a) (espanhol), laisser, finire, couple, relation, violent(e) (francês).

O critério de inclusão foi tratar do processo de término ou da dinâmica de ficar ou sair da relação. Os critérios de exclusão foram tratar de violência em relações não amorosas e ter sido publicado antes de 2001 (ano de publicação do artigo mais recente da revisão de Anderson \& Saunders, 2003). Uma exceção foi feita em relação a um artigo de 1999 que não havia sido inserido na revisão anterior. Ademais, incluíram-se na revisão artigos acessados por meio de autores que investigam essa temática.

Para fins de análise, os catorze artigos foram separados em estudos com métodos quantitativos e estudos com métodos qualitativos, havendo, para esses dois grupos, critérios de análise cujo interesse foi comum a ambos os tipos de método e outros específicos dos estudos quantitativos. Os critérios comuns a ambos os grupos foram referentes ao método (delineamento, amostra, recrutamento da amostra, procedimentos de coleta e instrumentos), ao(s) modelo(s) teórico(s) adotado(s), aos tipos de relacionamentos englobados (se namoro, casamento, relacionamento estável ou outros) e aos resultados encontrados. As informações específicas para análise dos estudos quantitativos foram: variáveis independentes, variáveis mediadoras e variáveis dependentes.

\section{Resultados e discussão}

Foram encontrados catorze artigos publicados entre 1999 e 2015. A pesquisa gerou doze estudos em inglês e um em português. Os países de atuação dos autores foram Estados Unidos (onze artigos), Brasil (um), Austrália (um) e Suécia (um). Os dados serão apresentados com base nos tipos de estudo: quantitativo ou qualitativo.

\section{Estudos quantitativos}

Dos catorze artigos incluídos nesta revisão, sete utilizaram métodos quantitativos. A análise do delineamento dos estudos revelou que todos adotaram modelos observacionais, em sua maioria com recortes transversais (Alexander, Tracy, Radek, \& Koverola, 2009; Choice \& Lanke, 1999; Pape \& Arias, 2000; Shorey, Tirone, Nathanson, Handsel, \& Rhatigan, 2013; Shurman \& Rodriguez, 2006). Tal delineamento tem como vantagens o melhor ajuste às possibilidades financeiras da pesquisa, além da economia de tempo. Por outro lado, permite observar um recorte cronológico limitado, implicando delineamento mais frágil quando se quer atribuir relação de antecedência e sucessão de eventos. Ademais, a complexidade e a volatilidade da dinâmica de abandonar e retomar a relação não pode ser capturada por esse tipo de método. Entre as outras pesquisas, duas apresentaram recorte longitudinal (Bell et al., 2007; Edwards, Gidycs, \& Murphy, 2015), que tem maior poder de estabelecer relações de antecedência e sucessão entre os eventos.

Dentre as escalas adotadas, observa-se que a versão revisada da Escala de Táticas de Conflito (Conflict Tactis Scales - revised - CTS-2), de Straus, Hamby, Boney-McCoy e Sugarman, 1996, vem sendo relevante para os estudos do campo. O uso de um mesmo instrumento em estudos distintos apresenta a vantagem de permitir comparações entre os dados de diferentes pesquisas. Por outro lado, esse instrumento se restringe ao acesso da violência em situações de conflito. Embora essa seja uma dimensão importante da violência, ela não encerra o fenômeno, uma vez que outras formas de violência, especialmente aquela de caráter simbólico (Bourdieu, 2019), não costuma gerar conflito e, ainda quando isso ocorre, a violência simbólica é comumente ignorada da situação. Esse termo é utilizado para definir formas de violência que são legitimadas nas relações e estruturas sociais, sendo aceitas culturalmente (Bourdieu, 2019). Por esse motivo, elas passam despercebidas no senso-comum e mesmo na avaliação de muitos especialistas. Seus impactos, no entanto, são bastante devastadores, uma vez que criam hierarquias na maneira como as pessoas conseguirão acessar oportunidades e direitos. A violência de gênero e a violência racial, por exemplo, são formas de violência simbólica. No que concerne à violência doméstica, por exemplo, uma expressão significativa 
de violência simbólica diz respeito à sobrecarga das mulheres com o trabalho da casa e dos filhos. Embora seja naturalizada, aceita e ocorra na quase totalidade dos lares, essa desigualdade tem consequências significativas para as mulheres: representa prejuízos às possibilidades de carreira, lazer, descanso e aceitação no grupo social. Por conseguinte, tem grande potencial de danos à autonomia financeira, à saúde mental, ao desenvolvimento de habilidades de vida e até mesmo ao acesso à proteção (que com frequência é indevidamente negada pelos agentes públicos a mulheres que não desempenham esse papel a contento, risco que os homens não correm a despeito do grau de abandono em que possam deixar seus filhos, por exemplo). A falta de instrumentos que acessem essa dimensão representa enorme lacuna nos estudos sobre relacionamentos violentos. A título de exemplo, tome-se como contexto um conflito que se dê em razão de o homem se recusar a cuidar dos filhos para ela poder estudar. Em uma situação como essa, pode ocorrer de a ofensa ou o grito hostil partirem dela. No instrumento que se baseia em comportamentos em situações de conflito, registra-se o grito e a ofensa dela. No entanto a violência de gênero, que nega a ela a possibilidade de exercer direitos básicos de liberdade, autonomia e, mais grave, de estruturar sua vida para superar a violência naquele relacionamento, passa completamente despercebida por esse tipo de instrumento.

A primeira característica que se destaca quanto aos modelos teóricos adotados é a inserção crescente do modelo transteórico de mudança como referencial teórico nesses estudos. Das sete pesquisas quantitativas, três utilizam esse modelo como base de coleta e análise dos dados (Alexander et al., 2009; Shorey et al., 2013; Shurman \& Rodriguez, 2006). Anderson e Saunders (2003) haviam apontado para seu uso em estudos qualitativos e para a tendência, nesses, de considerar o caráter processual do abandono da relação. Esse entendimento parece ser, paulatinamente, absorvido nessas pesquisas quantitativas, que observaram como características demográficas e outras variáveis independentes de interesse se relacionavam com os estágios de mudança, trazendo, assim, a possibilidade de compreender, mesmo em estudos quantitativos, o abandono não como um ponto no tempo, mas como uma construção que passa por distintas etapas. As variáveis estudadas foram: estilos de apego, atribuição (de responsabilidade), depressão, desesperança, ansiedade e raiva (Shurman \& Rodriguez, 2006), características demográficas, estilo de apego, característica da violência, natureza do relacionamento e suporte social (Alexander et al., 2009), além de normas subjetivas e comprometimento (Shorey et al., 2013). Outro modelo teórico que teve adesão foi o modelo de investimento, usado em três investigações (Choice \& Lanke, 1999; Edwards et al., 2014; Shorey et al., 2013).

Os dados apontaram uma diversidade de variáveis investigadas ao longo de todos os estudos analisados (Tabela 1).
Tabela 1. Frequência com que cada variável foi estudada

\begin{tabular}{lc}
\hline \multicolumn{1}{c}{ Variável independente ou mediadora } & $N^{o}$ \\
\hline Características da violência & 3 \\
Normas subjetivas $^{\text {b }}$ & 3 \\
$\begin{array}{l}\text { Nível de investimento na relação (satisfação } \\
\text { com o relacionamento, qualidade das } \\
\text { alternativas e investimentos irrecuperáveis) }\end{array}$ & 2 \\
$\begin{array}{l}\text { Comprometimento } \\
\text { Estilos de apego }\end{array}$ & 2 \\
$\begin{array}{l}\text { Atribuição da responsabilidade } \\
\text { pela violência ao parceiro }\end{array}$ & 2 \\
$\begin{array}{l}\text { Recursos e barreiras estruturais (renda, } \\
\text { despesas, bens em comum) }\end{array}$ & 2 \\
$\begin{array}{l}\text { Comportamento planejado (intenções de deixar a } \\
\text { relação, atitudes e controle sobre o comportamento) }\end{array}$ & 2 \\
$\begin{array}{l}\text { Sentimentos (raiva, tristeza, } \\
\text { nervosismo, desesperança) }\end{array}$ & 2 \\
$\begin{array}{l}\text { Impactos na saúde mental (depressão e ansiedade) } \\
\text { Satisfação com o suporte social } \\
\text { Natureza do relacionamento }\end{array}$ & 1 \\
\hline
\end{tabular}

${ }^{\text {aPape \& Arias (2000) estudaram reações emocionais e atribuição }}$ de responsabilidade pela violência como variáveis mediadoras entre a severidade da violência e a intenção de deixar a relação. bhorey et al. (2013), além de estudarem comprometimento e normas subjetivas como variáveis independentes, verificaram se há mediação entre as duas variáveis.

Entre essas variáveis, aquelas para as quais se encontrou correlação com a intenção, a efetivação ou o processo de término do relacionamento de forma consistente entre os estudos ou com indicação considerável dentro de apenas um estudo (nos casos em que tenha sido estudada apenas uma vez) foram normas subjetivas ( $\mathrm{n}=3$; Choice \& Lanke, 1999; Shorey et al., 2013; Edwards et al., 2015), investimento na relação e comprometimento $(\mathrm{n}=3$, sendo que Choice e Lanke estudam as duas variáveis como construtos separados) (Choice \& Lanke, 1999; Shorey et al., 2013; Edwards et al., 2015), atribuição da responsabilidade pela violência ao agressor ( $\mathrm{n}=2$; Pape \& Arias, 2000; Shurman \& Rodriguez, 2006), recursos e barreiras estruturais $(n=2$; Choice \& Lanke, 1999; Alexander et al., 2009), comportamento planejado ( $\mathrm{n}=1$; Edwards et al., 2015) e sentimento de raiva $(\mathrm{n}=1$; Shurman \& Rodriguez, 2006). Normas subjetivas favoráveis ao término, atribuição de responsabilidade pela violência ao agressor, existência de recursos estruturais (ex.: ter renda própria), comportamento planejado e sentimento de raiva tiveram correlação positiva com o término da relação. Investimento na relação, comprometimento e existência de barreiras estruturais (ex.: não ter renda própria) tiveram correlação negativa com o término. Vale ressaltar que investimento na relação e comprometimento são definidos por fatores muito próximos nos artigos que estudam essas variáveis. 
Tais resultados revelam uma grande variedade de aspectos relacionados ao avanço rumo ao abandono da relação violenta, apontando para a considerável quantidade de determinantes que atuam nesse processo. Nesse sentido, aspectos do contexto social (normas subjetivas), do contexto estrutural (renda, ter onde morar etc.) e pessoais (investimento na relação, comprometimento, emoções, atribuição de responsabilidade) podem contribuir ou configurar barreiras para o processo de superação da violência.

As variáveis cuja correlação se mostrou inexistente foram as características da violência e a satisfação com o suporte social (Alexander et al. 2009). Sobre a primeira, Anderson e Saunders (2003) já haviam apontado para esse resultado entre os estudos, destacando, no entanto, a falta de padronização dos conceitos e classificação. A persistência de achados nesse sentido, no entanto, reforça a evidência de que a severidade da violência não guarde relação com o processo de término. Sobre a segunda, as autoras discutem que a rede social, embora satisfatória, pode ser dividida com o agressor e não oferecer suporte para a saída da relação, ainda que proteja quanto à severidade da violência. Dessa forma, avaliam que a variabilidade e amplitude da rede de suporte é mais importante que a satisfação com ela.

Os resultados referentes ao estilo de apego foram inconsistentes entre os dois estudos que consideraram essa variável. Alexander et al. (2009) encontraram o apego preocupado como associado aos estágios iniciais do modelo transteórico de mudança. Shurman e Rodriguez (2006), por sua vez, encontraram relação entre esse estilo de apego e a prontidão para mudança entre os estágios. É possível que diferenças das amostras tenham gerado tais resultados, especialmente considerando que Shurman e Rodriguez trabalharam com mulheres que estavam em abrigos ou residências de apoio transitórias, sendo comum, nesses casos, a associação com outros fatores de vulnerabilidades, especialmente os referentes à escassez de renda e de suporte social. Se por um lado esse aspecto traz viés considerável ao perfil da amostra, por outro, destaca as características de um grupo em situação de muita vulnerabilidade e, por conseguinte, cujas necessidades precisam ser priorizadas.

Um aspecto importante a ser observado é a grande diversidade de variáveis investigadas diante do reduzido número de artigos encontrados, de forma que cada uma foi estudada em, no máximo, três pesquisas. Da mesma forma, não existe aprofundamento nas características relacionadas a nenhuma variável de interesse, tampouco segmentação entre grupos distintos. Esse quadro sinaliza para o caráter multicausal dos aspectos relacionados ao abandono dos relacionamentos violentos, o que gera hipóteses muito variadas advindas de observações em contextos específicos e de teorias distintas.

As variáveis dependentes estudadas foram a intenção de abandono da relação, a permanência ou saída da relação e os estágios do modelo transteórico de mudanças tendo como foco a saída definitiva da relação. É importante ressaltar que, embora possam ser pensadas em um continuum, elas não necessariamente falam de gradações do mesmo fenômeno, uma vez que a intenção de deixar o relacionamento nem sempre se concretiza e que estar fora dele não significa que essa condição irá se manter. Os dois aspectos, contudo, estão englobados ao se pensar o abandono como um processo, como ocorre nos estágios do modelo transteórico de mudanças.

A análise das características do recrutamento das amostras revelou quatro ambientes ou estratégias: recrutamento comunitário $(n=3)$, entre estudantes $(n=2)$, em centros de atendimento a vítimas ou agressores $(n=3)$ e em abrigos $(n=3)$. Sobre essas formas, é importante fazer algumas considerações. É possível que nenhuma delas consiga recrutar casos em que a pessoa agredida está isolada da rede de serviços de atenção à violência ou inibida em tornar conhecida a situação de agressão, seja por razões individuais (vergonha, falta de acesso), por aquelas referentes às características da violência (medo, grave ameaça) ou por estarem em situação de grande exclusão social (pessoas em situação de rua ou não alfabetizadas, por exemplo). Tais condições acarretam maior nível de vulnerabilidade e estresse, e amostras que as englobem podem gerar resultados com vistas a informar sobre necessidades não endereçadas nos serviços de atendimento existentes atualmente. Tendo em conta que algumas dessas condições podem ser mais comuns do que se imagina e que ela implica graves riscos, estratégias que permitam o acesso a elas no contexto da pesquisa são importantes. Algumas alternativas residem na recorrência a contextos existentes em políticas públicas, como aquelas de acesso facilitado (por exemplo, o Ligue 180, no Brasil), de busca ativa (como casos identificados no contexto dos atendimentos das equipes do Saúde da Família ou da Polícia Militar) ou na realização de estudos de caso (uma vez que será pouco provável o número de participantes que permitam outros delineamentos) em situações identificadas em serviços não direcionados ao atendimento de casos de violência, por exemplo, nos hospitais gerais.

Outro limitador considerável consiste no poder de generalização e nas limitações à interpretação dos dados com amostras recrutadas em abrigos. Em geral, as pesquisas não trouxeram informações detalhadas acerca da situação de vulnerabilidade social (exceto aquelas relativas à renda) e da existência ou não de suporte social e familiar. Por outro lado, o público atendido por esse tipo de serviço costuma englobar pessoas com alto nível de vulnerabilidade social, dificuldades financeiras e rede de suporte social escassa e inefetiva. Nesse sentido, dados como os encontrados por Shurman e Rodriguez (2006), de que depressão foi preditor do estágio de manutenção no modelo transteórico de mudanças, podem estar mais relacionados a essas características da amostra do que ao estágio de mudança em si, além de que talvez não possam ser generalizáveis para outros grupos. Apesar disso, 
os estudos com mulheres vivendo em abrigos são extremamente importantes justamente por lançarem luz a uma parcela muito vulnerável da população. Todavia é preciso fazer ressalvas a seu poder de generalização. Esse aspecto reforça o direcionamento de que os grupos não sejam tratados como homogêneos, mas que se façam recortes de acordo com suas especificidades.

Sobre os tipos de relacionamentos pesquisados, apenas um estudo dedicou-se exclusivamente às relações pré-matrimoniais (Choice \& Lanke, 1999). Outros três envolveram, ainda que de forma minoritária, relações de namoro na amostra (Bell et al., 2007; Edwards et al., 2015; Shurman \& Rodriguez, 2006). No entanto eles não segmentam suas análises em função do tipo de relacionamento. Dessa forma, não é possível esclarecer se existem particularidades no processo de abandono desse tipo de relação quando comparados a casamentos ou relações em que há coabitação. Essa permanece uma lacuna na literatura que limita as formas de intervenção para as pessoas em relacionamentos de namoro e apresenta um grande custo de oportunidade no que diz respeito à prevenção.

Entre os estudos quantitativos, apenas um (Bell et al, 2007) se debruçou sobre o período que sucede o término da relação, tendo investigado bem-estar e exposição à violência diante de diferentes desfechos que se seguiram à condição de estar separada do parceiro no início do estudo (manter separação, retomar a relação de forma estável ou manter separação e retomar relação alternadamente). Considera-se esse estudo de especial relevância por lançar luz nos acontecimentos posteriores ao término da relação, apontando para o fato de que nem todas as experiências de rompimento indicam um mesmo fenômeno e abrindo um caminho de pesquisa precioso no campo quantitativo para a geração de conhecimento que permita o delineamento de políticas públicas e de estratégias de atendimento mais adequadas. Anderson e Saunders (2003), em sua revisão, identificaram a escassez de estudos sobre o período que sucede o fim da relação como uma lacuna importante. Desde então, houve pouco avanço nesse ponto nos estudos quantitativos.

\section{Estudos qualitativos}

Dos catorze artigos incluídos nesta revisão, sete adotaram métodos qualitativos. No que diz respeito aos aspectos do método, a entrevista se mostrou como procedimento mais usual de coleta de dados, sendo que, à exceção de dois, todos os outros lançaram mão desse recurso (Cluss et al., 2006; Few \& Rosen, 2005; Meyer, 2012; Murta, Ramos, Cangussú, Tavares, \& Costa, 2014; Wiklund, Malmgren-Olsson, Bengs \& Öhman, 2010). Anderson (2003) aplicou a técnica Delphi, que visa à coleta de dados com especialistas, e Edwards et al. (2012) utilizaram respostas escritas a perguntas abertas. A entrevista enquanto estratégia de coleta de dados apresenta a vantagem de ser acessível, com maior facilidade para o pesquisador. Por outro lado, a quase inexistência de outras estratégias de coletas de dados pode limitar o acesso a determinados tipos de informações que não são acessíveis por meio de entrevista.

Grande parte dos estudos utilizou o modelo transteórico de mudanças, seguindo aquilo que já havia sido observado por Anderson e Saunders (2003). Dos sete estudos, quatro lançaram mão dele, sendo um relacionado à análise de sua adequação ao fenômeno (Anderson, 2003), um partindo desse referencial e questionando-o posteriormente - gerando o Modelo de Prontidão Psicossocial - (Cluss et al., 2006), um utilizando-o para analisar o avanço nos estágios em um intervalo de dois meses (Edwards et al., 2012) e o último aprofundando as análises na identificação de estratégias que colaboram para a passagem de um estágio ao outro (Murta et al., 2014).

Um aspecto relevante nesses estudos, ainda, consiste na frequência com que se dedicam a analisar a adequação ou geração de modelos. Todos eles, em algum grau, envolvem uma dessas vertentes. Além das verificações já citadas em torno do modelo transteórico de mudanças, identifica-se a adequação à teoria da escolha racional e a geração de novos modelos: modelo de prontidão psicossocial (Cluss et al., 2006) e modelo conceitual de vulnerabilidade (Few \& Rosen, 2005). Wiklund et al. (2010), embora não proponham novo modelo, adotam etapas e constroem uma linha cronológica para o enfrentamento da violência (Wiklund et al., 2010). Todos os modelos incluem aspectos relativos às barreiras e aos recursos de enfrentamento internos e externos que permitem chegar ao término da relação e à manutenção dessa condição.

O modelo de prontidão psicossocial (Cluss et al., 2006) é composto por três fatores internos - percepção de suporte social, autoeficácia e tomada de consciência que são situados ao longo de um continuum que vai da manutenção total do status quo à superação da violência. Dois fatores externos - suporte social e condições situacionais (ex.: ter renda, ter casa etc.) - empurram os fatores internos para um lado ou outro do continuum.

O modelo conceitual de vulnerabilidade (Few \& Rosen, 2005) é caracterizado por duas dimensões: vulnerabilidade relacional (crenças sobre si na relação com os outros ou sobre o que é aceitável em um relacionamento) e vulnerabilidade estrutural (estressores advindos de mudanças tanto inerentes ao ciclo de vida como inesperadas, ou da falta de que se atinjam expectativas sociais ligadas ao status de relacionamento). A representação do modelo é feita em plano cartesiano. Essas duas dimensões estão imersas no contexto sociocultural.

A linha cronológica do enfrentamento da violência (Wiklund et al., 2010) não chega a ser proposta pelas autoras como modelo teórico, contudo apresenta estruturas de análise comuns para os dois casos estudados, tendo como base a linha cronológica dos eventos, diferenciando 
distintas fases rumo ao abandono da relação e ao enfrentamento das consequências da violência após o seu término. Ela inicia com "estar sob controle de um namorado violento", passando por "lutando para parar a violência e terminar a relação", "voltar a andar com os próprios pés e fazer um novo começo" e terminando com "deixar a relação e lidar com as consequências da violência". Para cada ponto desse processo, os desafios e comportamentos adotados são descritos.

No escopo dos estudos qualitativos, ainda, algumas das estratégias às quais as participantes recorreram no processo de término da relação foram identificadas. Auto-observação, suporte social, autocuidado, busca de informações, teste de soluções (ex.: terminar e retomar, tentar acabar com a violência dentro da própria relação), avaliação de riscos para terceiros, controle de estímulos, análise de riscos e benefícios, regulação de emoções, empoderamento (Murta et al., 2014). Após o término, trazer à consciência os aspectos relacionados às emoções negativas ligadas à relação e os próprios esquemas disfuncionais que operam nas relações amorosas, manejar situações de risco, dedicar atenção a necessidades de outros campos da vida, análise de prejuízos e ganhos, autovalorização e dedicar-se a novos interesses foram as estratégias mapeadas (Murta et al., 2014). Anderson (2003) classificou as estratégias de acordo com os processos de mudança previstos no modelo transteórico de mudanças.

No que concerne ao recrutamento da amostra, as características foram distintas em alguns pontos quando comparadas aos estudos quantitativos. Utilizaram-se como ambientes/grupos de recrutamento: serviços de saúde não relacionados diretamente à violência $(\mathrm{n}=3)$, abrigos para mulheres vítimas de violência $(n=2)$, lideranças comunitárias no combate à violência nos relacionamentos que passaram por esse tipo de experiência $(\mathrm{n}=1)$, estudantes universitárias $(\mathrm{n}=1)$, serviços de atendimento a vítimas de violência $(\mathrm{n}=1)$, além de amostra de conveniência $(\mathrm{n}=1)$.

Embora não tenha contado com recrutamento comunitário, a inclusão significativa do recrutamento em serviços de saúde não relacionados diretamente à violência aumenta as chances de acesso a pessoas que não buscam os serviços direcionados a essa problemática ou que não estão dispostas a revelar a condição de abuso na relação espontaneamente. Esses espaços são considerados privilegiados no acesso a vítimas de violência, pois, com maior frequência, elas os procuram com queixas que não relacionam de forma explícita à violência (ex.: dores, lesões, sintomas depressivos). Uma abordagem cuidadosa do profissional de saúde ou do pesquisador, contudo, pode ser um importante passo rumo ao melhor enfrentamento do problema, além de poder contribuir para a geração de novos conhecimentos (Zuma, Mendes, Cavalcanti, \& Gomes, 2014). Esse aspecto, mais uma vez, revela a importância do uso de delineamentos qualitativos na elucidação de situações nas quais o acesso aos casos é muito difícil, limitando seu número.
Por fim, a abordagem do período pós-término da relação, apontado como uma lacuna na revisão anterior de Anderson e Saunders (2003), foi contemplada em algum grau em seis dos sete estudos qualitativos (Anderson, 2003; Cluss et al., 2006; Few \& Rosen, 2005; Meyer, 2012; Murta et al., 2014; Wiklund et al., 2010). Dois trataram das estratégias de enfrentamento às dificuldades e barreiras à manutenção do término da relação (Anderson, 2003; Murta et al., 2014), um apontou para estratégias de proteção desenvolvidas após o fim da relação e impactos na saúde (Wilklund et al., 2010), um focou na discussão das decisões informadas pela avaliação de risco maior para si e para os filhos, no caso de abandono da relação, e as estratégias de planejamento necessárias para sair dessa relação (Meyer, 2012) e dois o trataram por meio da inserção de aspectos facilitadores e barreiras nos modelos gerados para a compreensão do fenômeno (Cluss et al., 2006; Few \& Rosen, 2005). Se por um lado se observa preocupação com o período após o término nos estudos qualitativos, seja em relação aos impactos ou às estratégias e barreiras experimentadas, por outro, o alvo e a referência permanecem em uma única relação e em seus impactos. Considerando que as experiências com relacionamentos violentos não necessariamente se restringem a uma única relação (Martsof et al., 2012), resta como lacuna a necessidade de estudos que se aprofundem na transição entre relacionamentos e cuja referência não esteja em apenas um deles.

\section{Considerações finais}

Identificaram-se, nessa revisão, algumas características da produção de artigos sobre o tema do abandono de relacionamentos violentos entre 1999 e 2015. Os estudos quantitativos analisados foram marcados pelo início da inserção da dimensão processual por meio do modelo transteórico de mudanças na explicação do abandono de relações violentas, a grande diversidade de variáveis, a pouca diferenciação quando da análise dos dados entre as relações de namoro e casamento, e a limitação na variedade de recrutamento da amostra. Os estudos qualitativos, por sua vez, tiveram como características a adoção marcante da dimensão processual do abandono da relação violenta, a busca pela adequação e geração de modelos de entendimento do fenômeno, a variedade de recrutamento da amostra, com inclusão de grupos recrutados em serviços de saúde não relacionados diretamente à violência, e a consideração de aspectos relacionados ao período pós-término da relação.

Os artigos qualitativos foram unânimes em lançar mão de teorias que compreendem o abandono como um processo, seja por meio do modelo transteórico de mudança, seja pela perspectiva da geração de outras teorias, como o modelo de prontidão psicossocial (Cluss et al., 2006) ou o modelo conceitual de vulnerabilidade (Few \& Rosen, 2005). No que diz respeito aos estudos quantitativos, a compreensão do caráter processual do fenômeno consiste 
mais especificamente na adoção do modelo transteórico de mudanças. Esses aspectos confirmam a análise da revisão de Anderson e Saunders (2003), que apontaram para a adoção da compreensão do abandono como processo nos estudos qualitativos produzidos até então. Nos últimos anos, portanto, essa tendência foi observada também nos estudos quantitativos.

A produção de conhecimento sobre o período após o término de relações íntimas violentas foi indicada por Anderson e Saunders (2003) como uma lacuna. Aparentemente, embora tenha havido avanços nesse campo, eles foram tímidos. Entre os estudos qualitativos, a investigação de aspectos relacionados ao período após o término da relação centrou-se no período que se seguiu imediatamente ao término (ou a manutenção do fim da relação). Entre os estudos quantitativos, apenas dois mencionam temas relacionados a esse momento em suas discussões, sem, contudo, abordá-los de forma central, e apenas um tem como foco a elucidação das condições vivenciadas nesse período e seus impactos sobre o bemestar e a vivência de violência.

As implicações dos resultados para a prática profissional apontam para estratégias preciosas a serem adotadas pelos serviços de atendimento. A importância das normas subjetivas como facilitadoras (ou barreiras) do término ressalta a necessidade de que as intervenções não sejam focadas exclusivamente na vítima ou no autor da violência, mas englobem outras pessoas importantes. No contexto macro, a abordagem do tema nos meios de comunicação com foco na quebra de preconceitos e disseminação de ideias que favoreçam a proteção e o suporte por pares ou familiares a pessoas que sofrem violência apresenta grande potencial de contribuir para suporte social mais adequado, o que tende a gerar impactos positivos no término da relação. O investimento na qualidade das relações de suporte também ganha destaque diante dos resultados que apresentaram o investimento na relação como fator relacionado com o término. Sendo a qualidade das alternativas parte do construto que define investimento, relações sociais de amizade positivas tendem a aumentar a avaliação da alternativa de terminar um relacionamento e permanecer sem parceiro. Nesse sentido, pessoas que contam com suporte social para outras esferas de sua vida também contam com maior condição de suporte emocional para enfrentar a difícil tarefa de terminar e permanecer fora de uma relação.

A ênfase na qualidade das alternativas à relação traz outro desdobramento para os serviços de atendimento a vítimas de violência: a criação de espaços de convivência baseados em atividades de lazer e socialização que não abordem diretamente a violência pode ser uma estratégia importante no seu combate ao fomentar relações sociais e ampliar repertórios. Ainda, tais estratégias contam com a vantagem de serem mais atraentes ao público que ainda tem dificuldade em se perceber em condição de violência.
A necessidade de endereçar barreiras externas estruturais ao término mais uma vez é evidente a partir dos resultados apresentados. Tais barreiras coincidem com contextos de vulnerabilidade social, especialmente os relativos à pobreza. Nesse sentido, iniciativas relativas à geração de renda, à habitação (especialmente em caráter emergencial) e ao atendimento das necessidades básicas em caráter emergencial (a exemplo dos auxílios emergenciais previstos no Sistema Único de Assistência Social - Suas) demandam atenção dos serviços. Aparentemente, abordagens profissionais interdisciplinares que enderecem $\mathrm{o}$ atendimento às necessidades de ordem prática (que atuam como barreiras estruturais externas) e àquelas que perpassam as questões psicossociais (de ordem interna) apresentam maior potencial de efetividade.

Ainda sobre as barreiras estruturais, ressalta-se a importância da existência de marco legal de proteção à mulher, como ocorreu no Brasil com a Lei Maria da Penha, que inaugurou uma nova era no atendimento às situações de violência no âmbito doméstico e familiar. A existência de políticas de Estado propicia a acentuada diminuição de barreiras externas, uma vez que retira da esfera individual e da família o ônus exclusivo de lidar com a violência e insere proteções legais que instrumentalizam a busca de direitos, o fazer valer a vontade individual acerca do fim da relação e criam, no longo prazo, mudanças sociais favorecedoras do fim da violência.

Os resultados desta revisão devem ser interpretados à luz de suas limitações. Dentre estas, apontam-se a dificuldade de replicação dos processos de busca, o que reduz seu caráter de sistematização. Ademais, salienta-se o fato de que os dados foram analisados por apenas uma autora, sem verificação de concordância entre codificadores, o que limita a confiabilidade das análises. Por fim, o contexto epistemológico não constou entre os critérios de análise dos estudos identificados, o que pode ser sanado em novas revisões.

Algumas lacunas são identificadas na literatura a partir desta revisão. Em primeiro lugar, observa-se a necessidade de mais pesquisas que tenham como foco o período pós-separação, lacuna que já havia sido apontada na revisão anterior e para a qual houve poucos avanços desde então. Esse aspecto tem especial importância ao se levar em conta que as separações nem sempre são definitivas e, em alguns casos, não suficientes para gerarem bem-estar. Para além do período que sucede imediatamente ao término, a transição para novas relações tem sido negligenciada pelos estudos científicos. Tendo em conta que o interesse social no esclarecimento das questões relativas ao término de relacionamentos violentos não é apenas de que as pessoas terminem relações pontuais, mas que vivam livres da violência nos relacionamentos íntimos, a maior compreensão dos processos de transição para novos relacionamentos é urgente e poderá permitir o aprimoramento da oferta de suporte às pessoas que abandonam relacionamentos violentos. 
Outro aspecto que carece de esclarecimento concerne à geração de maior conhecimento acerca de pessoas e grupos que não acessam os serviços direcionados à atenção à violência ou sequer revelam essa condição. Tal grupo se encontra em condição de extrema vulnerabilidade, o que ressalta a necessidade de busca ativa por esses sujeitos e demanda, prioritariamente, o uso de métodos qualitativos, tanto por permitirem a compreensão aprofundada de realidades individuais como em razão da dificuldade de acesso a essas pessoas que não solicitam ajuda. Estratégias que recorram à internet, tanto para pesquisa como para intervenção, também se apresentam promissoras.

Sob o prisma da prevenção, destaca-se a necessidade de maior conhecimento dos processos iniciais que favorecem o término como estratégia preciosa de enriquecimento dos programas de prevenção indicada, em especial nas relações de namoro nas quais a violência se mostre inicialmente. A entrada em relacionamentos violentos, ainda, merece atenção a partir dessa perspectiva. Compreender como as pessoas entram em relações violentas pode apontar para estratégias significativas para a prevenção universal (aquela direcionada a toda a população).
Por fim, ressalta-se a necessidade de estratificação dos dados relativos a relações de namoro e de casamento/ relação estável, tanto em razão das especificidades de necessidades de intervenção em cada tipo de relação como pelo grande potencial de geração de intervenções preventivas existente na maior compreensão dos processos envolvidos na relação de namoro. $\mathrm{O}$ tratamento dos dados como caracterizando um mesmo grupo pode estar escondendo especificidades relevantes e nublando as análises. Ademais, ressalta-se a importância de estudos que considerem a estratificação de grupos e perfis, adotando variáveis moderadoras, dada a grande variedade de condições internas e externas às quais as pessoas estão sujeitas no contexto de abandono de relações violentas e o potencial de esclarecimento acerca da divergência de resultados a partir de dados advindos de amostras distintas. Vulnerabilidades relacionadas à pobreza se mostram mais evidentes nos estudos, porém, outras questões sociais como as concernentes a raça, orientação sexual, ter uma deficiência, por exemplo - cuja intersecção se relaciona com a vivência de relacionamentos violentos, não devem ser ignoradas e poderão ser mais bem estudadas em pesquisas futuras. Ainda, diferenças entre relações de namoro e casamento valem ser mais bem exploradas.

\title{
Leaving a violent intimate relationship: a literature review
}

\begin{abstract}
This article analyzes the advances and limitations in the scientific production regarding the process of leaving violent intimate relationships. For that, articles addressing leaving violent relationships or the stay/leave process as the main theme, written in English, Portuguese, Spanish, and French, were searched in Periódicos Capes and SciELO Web searches. The search resulted in 14 articles published between 1999 and 2015 - half with a quantitative design and the other half with a qualitative design. Investment, commitment, subjective norms, responsibility attribution, anger, and structural barriers and facilitators were correlated with the leaving process. These studies also understood the end of relationship as a process. Qualitative studies proposed new models, focused on strategies that enable the leaving process, and considered the post-separation period in the analysis. Thus, this study provides recommendations for professional practice and further research on the theme.
\end{abstract}

Keywords: domestic violence, intimate partner violence, violence against women.

\section{Mettre fin aux relations intimes violentes : une revue de la littérature}

Résumé : Cet article analyse les avances et les limites de la production scientifique sur le processus de rompre des relations intimes violentes. On a mené une recherche dans les bases de données Portal Periódico Capes et SciELO, avec des mots-clés en anglais, français, espagnol et portugais. Les études discutant la fin des relations amoureuses violentes ou les aspects de la décision de rester ou de partir ont été incluses. On a trouvé 14 études publiées entre 1999 et 2015, dont la moitié avait une conception quantitative et l'autre moitié, qualitative. Dans les sept études quantitatives, les variables suivantes ont montré une corrélation avec la progression vers la fin de la relation : niveau d'investissement, engagement, normes subjectives, attribution de la responsabilité de l'agression à l'agresseur, colère, obstacles et facilitateurs structurels. Pour les sept études qualitatives, on a observé : la proposition de nouveaux modèles de compréhension, la plus grande variabilité dans le recrutement des échantillons et la prise en compte de la période post-séparation dans les analyses. Des recommandations pour la pratique professionnelle et la recherche sont discutées.

Mots-clés : violence domestique, violence envers le partenaire intime, violence contre les femmes. 


\section{Término de relaciones de pareja violentas: una revisión de la literatura}

Resumen: Este artículo analiza los avances y las limitaciones en la producción científica sobre el proceso de término de relaciones de parejas violentas. Se realizaron búsquedas en las bases de datos Portal de Periódicos Capes y SciELO con entradas en inglés, español, francés y portugués. Los criterios de inclusión fueron tratar del término de relaciones de pareja violentas o de aspectos de la decisión de quedarse versus dejar. Se incluyeron 14 estudios publicados entre 1999 y 2015, los cuales la mitad fue de enfoque cuantitativo, y la otra mitad, cualitativo. Estas variables tuvieron correlación con el avance al término en los estudios cuantitativos: nivel de inversión, compromiso, normas subjetivas, atribuir responsabilidad al agresor por la agresión, sentimiento de rabia, barreras y facilitadores estructurales. Para los siete estudios cualitativos se observó: la propuesta de nuevos modelos de comprensión, la variabilidad de reclutamiento de los muestreos y la consideración del período que sigue la separación en los análisis. Se discuten recomendaciones para la práctica profesional e investigación.

Palabras clave: violencia doméstica, violencia de pareja, violencia contra la mujer.

\section{Referências}

Akoenski, T. D., Koehler, J. A., Lösel, F., \& Humphreys, D. K. (2013). Domestic violence perpetrator programs in Europe, part II: a systematic review of the state of evidence. International Journal of Offender Therapy and Comparative Criminology, 57(10), 1206-1225. doi: 10.1177/0306624X12468110

Alexander, P. C. Tracy, A., Radek, M., \& Koverola, C. (2009). Predicting stages of change in battered women. Journal of Interpersonal Violence, 24(10), 1672-1772. doi: 10.1177/0886260509331494

Anderson, C. (2003). Evolving out of violence: an application of the transtheoretical model of behavioral change. Research and Theory for Nursing Practice: An International Journal, 17(3), 225-240.

Anderson, D. K., \& Saunders, D. G. (2003). Leaving an abusive partner: an empirical review of predictors, the process of leaving and psychological wellbeing. Trauma, Violence and Abuse, 4(2), 163-191. doi: 10.1177/1524838002250769

Bell, M. E., Goodman, L. A., \& Dutton, M. A. (2007). The dynamics of staying and leaving: implications for battered women's emotional well-being and experiences of violence at the end of a year. Journal of Family Violence, 22(6), 413-428. doi: 10.1007/s10896-007-9096-9

Bourdieu, P. (2019). A dominação masculina. Curitiba, PR: Bertrand Brasil.

Brasil. (2006). Lei $n^{o}$ 11.340, de 7 de agosto de 2006. Cria mecanismos para coibir a violência doméstica e familiar contra a mulher. BRASIL. Recuperado de https://bit.ly/3tgPE1c

Choice, P., \& Lanke, L. K. (1999). Stay/leave decisionmaking processes in abusive dating relationships. Personal Relationships, 6(3), 351-367. doi: 10.1111/ j.1475-6811.1999.tb00197.x

Cluss, P. A., Chang, D. C., Hawker, L., Scholle, S. H., Dado, D., Buranosky, R., . . Goldstrohm, S. (2006). The process of change for victims of intimate partner violence: support for a psychosocial readiness model. Women's Health Issues, 16, 262-274. doi: 10.1016/j.whi.2006.06.006
DataSenado. (2011). Violência doméstica e familiar contra a mulher. Brasília, DF: Senado Federal.

Edwards, K. M., Gidycz, C. A., \& Murphy, M. J. (2015). Leaving an abusive dating relationship: a prospective analysis of the investment model and theory of planned behavior. Journal of Interpersonal Violence, 30(16), 2908-2927. doi: 10.1177/0886260514554285

Edwards, K. M., Murphy, M. J., Tansill, E. C., Myrick, C., Probst, D. R., Corsa, R., . . . Gidycs, C. A. (2012). A qualitative analysis of college women's leaving processes in abusive relationships. Journal of American College Health, 60(3), 204-210. doi: 10.1080/07448481.2011.586387

Feder, L., \& Wilson, D. B. (2005). A meta-analytic review of court mandated batterer intervention programs: can courts affect abusers' behavior? Journal of Experimental Criminology, 1(2), 239-262. doi: 10.1007/s11292-005-1179-0

Feder, L., Wilson, D. B., \& Austin, S. (2008). Court-mandated interventions for individuals convicted of domestic violence. Campbell Systematic Reviews, 12, 1-46.

Few, A. L., \& Rosen, K. H. (2005). Victims of chronic dating violence: how women's vulnerabilities link to their decisions to stay. Family Relations, 54(2), 265-279. doi: 10.1111/j.0197-6664.2005.00021.X

Martsof, D. S., Draucker, C. B., Stephenson, P. L., Cook, C. B., \& Heckman, T. A. (2012). Patterns of dating violence across adolescence. Qualitative HealthResearch, 22(9), 1271-1283. doi: 10.1177/1049732312449388

Meyer, S. (2012). Why women stay: a theoretical examination of rational choice and moral reasoning in the context of intimate partner violence. Australian \& New Zealand Journal of Criminology, 45(2), 179-193. doi: $10.1177 / 0004865812443677$

Murta, S. G., Ramos, C. E. P. L., Cangussú, E. D. A., Tavares, T. N. G., \& Costa, M. S. F. (2014). Desenvolvimento de um website para prevenção à violência no namoro, abandono de relações íntimas abusivas e apoio aos pares. Contextos Clínicos, 7(2), 118-132. 
Pape, K. T., \& Arias, I. (2000). The role of perceptions and attributions in battered women's intentions to permanently end their violent relationships. Cognitive Therapy and Research, 24(2), 201-214. doi: 10.1023/A:1005498109271

Prochaska, J. O., DiClemente, C. C., \& Norcross, J. C. (1992). In search of how people change. Applications to addictive behaviors. American Psychologist, 47(9), 1102-1114.

Secretaria Especial de Políticas para as Mulheres. (2012). Central de Atendimento à Mulher - Ligue 180: Dados consolidados - 2012. Brasília, DF: Presidência da República. Recuperado de https://bit.ly/3A6KnNu

Shorey, R. C., Tirone, V., Nathanson M., Handsel, V. A., \& Rhatigan, D. L. (2013). A preliminary investigation of the influence of subjective norms and relationship commitment on stages of change in female intimate partner violence victims. Journal of Interpersonal Violence, 28(3), 621-642. doi: 10.1177/0886260512455520

Shurman, L. A., \& Rodriguez, C. M. (2006). Cognitiveaffective predictors of women's readiness to end domestic violence relationships. Journal of Interpersonal Violence, 21(11), 1417-1439. doi: 10.1177/0886260506292993
Straus, M. A., Hamby, S. L., Boney-McCoy, S., \& Sugarman, D. B. (1996). The revised Conflict Tactics Scale (CTS2): development and preliminary psychometric data. Journal of Family Issues, 17(3), 283-316. doi: 10.1177/019251396017003001

Wiklund, M., Malmgren-Olsson, E., Bengs, C., \& Öhman, A. (2010). "He messed me up": Swedish adolescent girls' experiences of gender-related partner violence and its consequences over time. Violence Against Women, 16(2), 207-231. doi: 10.1177/1077801209356347

Zuma, C. E., Mendes, C. H. F., Cavalcanti, L. F., \& Gomes, R. (2013). Violência de gênero na vida adulta. In K. Njaine, S. G. Assis, \& P. Constantino (Eds.), Impactos da violência na saúde (pp. 149-196). Rio de Janeiro, RJ: Fiocruz.
Recebido: 13/03/2018

Revisado: 01/09/2020

Aprovado: 23/06/2021 